\title{
The Synthesis of New Neutron-Rich Heavy Nuclei
}

\author{
Walter David Loveland* \\ Chemistry Department, Oregon State University, Corvallis, OR, United States
}

All the known isotopes of the elements Fm through Og are neutron-deficient relative to $\beta$-stability. In this contribution, I discuss two methods of producing more $\mathrm{n}$-rich heavy nuclei, i.e., the use of radioactive nuclear beams (RNBs) and the use of multi-nucleon transfer (MNT) reactions. In the former case, I discuss recent studies of the interaction of ${ }^{39,46} \mathrm{~K}$ with ${ }^{181} \mathrm{Ta}$ and their implications for the synthesis of more $\mathrm{n}$-rich isotopes of $\mathrm{Bh}$ and $\mathrm{Hs}$. In the case of MNT reactions, I discuss recent results for the reaction of ${ }^{136} \mathrm{Xe}$ with ${ }^{208} \mathrm{~Pb},{ }^{204} \mathrm{Hg}+{ }^{198} \mathrm{Pt}$, and ${ }^{136} \mathrm{Xe}+{ }^{198} \mathrm{Pt}$. I compare measured distributions of the target-like fragments (TLFs) and projectile-like fragments (PLFS) with current models of MNT reactions.

Keywords: heavy ion collisions, multi-nucleon transfer reactions, GRAZING model, DNS model, ImQMD model, TDHF model, radioactive nuclear beams

PACS numbers: 25.70.Hi, 25.70.-z,24.10.Cn, 25.60.Je

\section{OPEN ACCESS}

Edited by:

Angela Bonaccorso,

Istituto Nazionale di Fisica Nucleare,

Sezione di Pisa, Italy

Reviewed by:

Kazuyuki Sekizawa,

Niigata University, Japan

Giuseppe Verde,

Istituto Nazionale di Fisica Nucleare,

Italy

*Correspondence:

Walter David Loveland

lovelanw@onid.orst.edu

Specialty section:

This article was submitted to

Nuclear Physics,

a section of the journal

Frontiers in Physics

Received: 16 November 2018

Accepted: 12 February 2019

Published: 08 March 2019

Citation:

Loveland WD (2019) The Synthesis of New Neutron-Rich Heavy Nuclei.

Front. Phys. 7:23.

doi: 10.3389/fphy.2019.00023

\section{INTRODUCTION}

All known isotopes of the elements Fm through Og are neutron deficient relative to $\beta$-stability. While there is some dispute as to the next proton magic number beyond 82 (with proponents of $Z$ $=114,120$, and 126) [1] there is little doubt that the next neutron magic number beyond $N=126$ is $N=184$. A clear-cut path to reach $N=184$ is not evident although one is encouraged by recent efforts that have reached $N=177$.

Two synthetic paths to making more neutron-rich super-heavy nuclei, the use of radioactive nuclear beams (RNBs) and the use of multi-nucleon transfer (MNT) reactions, seem to have emerged as the best approaches. The central issue for the use of RNBs for making new n-rich heavy nuclei is the available fluxes of n-rich projectiles $[2,3]$. The central issue for the use of MNT reactions to make new neutron-rich heavy nuclei is to understand the MNT processes well enough to justify vigorous experimental efforts.

There have been several papers dealing with these issues. Loveland has treated the use of RNBs to synthesize new n-rich heavy nuclei $[2,3]$. He concluded that RNBs are not useful for making the heaviest nuclei, but that there is a "window of opportunity" for making n-rich isotopes of elements 103-107 using FRIB. Wakhle et al. studied the reaction ${ }^{39,46} \mathrm{~K}+{ }^{181} \mathrm{Ta}$ [4] and concluded that Bh and $\mathrm{Hs}$ isotopes might be produced at rates $\sim 0.1$ atom/day at FRIB. Mun et al. [5] concluded that the radioactive beams of ${ }^{66,68,70} \mathrm{Ni}$ reacting with ${ }^{238} \mathrm{U}$ do not provide any advantage in producing neutron-rich isotopes of $\mathrm{Md}$ due to low beam intensities and reduced complete fusion cross sections. Zhu et al. concluded that the use of the RNB ${ }^{144} \mathrm{Xe}[6]$ could lead to increased production cross sections for heavy n-rich nuclei although the issue of production rates was not addressed. Zhu et al. [7] did conclude that ${ }^{144} \mathrm{Xe}$ could be used to produce neutron-rich nuclei with $\mathrm{Z}<\mathrm{Z}_{\text {target }}$. This conclusion is based on estimating [8] that ${ }^{144}$ Xe beam intensities at SPIRAL2 of $>10^{8} \mathrm{p} / \mathrm{s}$ will be available. It was suggested that these ${ }^{144} \mathrm{Xe}$ beams would be most effective in producing nuclei near the neutron closed shell $N=126$. Zhang et al. [9] concluded that ${ }^{145} \mathrm{Xe}+{ }^{208} \mathrm{~Pb}$ reactions could 
be used to produce unknown isotopes of $\mathrm{Pt}\left({ }^{209-212} \mathrm{Pt}\right)$ based upon similar estimates [8] of the intensities of ${ }^{145} \mathrm{Xe}$ beams at SPIRAL2. Li et al. [10] have pointed out the possibility of using ${ }^{22} \mathrm{O}$ in the ${ }^{22} \mathrm{O}+{ }^{244} \mathrm{Pu}$ reaction to make ${ }^{259-263} \mathrm{No}$.

Most of the interest in making new n-rich heavy nuclei has centered on the use of MNT reactions with stable beams. In experiments at GSI and LBNL [11-16], several studies were made of the possibilities of using MNT reactions to make new heavy nuclei. [A review of these data is found in [17]]. Production of n-rich trans-target nuclides up to Fm and $\mathrm{Md}$ was observed with cross sections of $\sim 0.1 \mu \mathrm{b}$. The basic problem was that the higher excitation energies that led to broader distributions of the trans-target nuclei caused the highly excited nuclei to fission, thereby restricting the use of these reactions to produce new n-rich heavy nuclei. Zagrebaev and Greiner [18-20] suggested that if MNT reactions were run near the barrier $\left[\sim 1.1 \mathrm{~V}_{\mathrm{b}}[21]\right]$ shell effects would be preserved and large transfers would occur. For the reaction of ${ }^{238} \mathrm{U}$ with ${ }^{248} \mathrm{Cm}$ at $1.1 \mathrm{~V}$, they predicted a net transfer of 30 nucleons from ${ }^{238} \mathrm{U}$ to ${ }^{248} \mathrm{Cm}$. The same calculational model when applied to the reaction of $\mathrm{E}_{\mathrm{c} . \mathrm{m} .}=750$ $\mathrm{MeV}{ }^{238} \mathrm{U}+{ }^{248} \mathrm{Cm}$ reproduced the previous measurements of Schadel et al. [14] and predicted the formation, at picobarn levels, of new n-rich isotopes of Sg.

\section{RNBs}

Why does one want to use RNBs for producing new n-rich heavy nuclei? Neutron-rich RNBs lead to the formation of more nrich products. These n-rich heavy nuclei have longer half-lives enabling more detailed atomic physics and chemistry studies. In Figure 1 [taken from [24]] I show the dependence of the half-life $\left(t_{1 / 2}\right)$ upon neutron number $\mathrm{N}$ for some typical even-even (e-e) heavy nuclei with $104 \leq \mathrm{Z} \leq 120$. One can see that the predicted half-lives increase logarithmatically with increasing neutron number showing maximum values for $N=184$. The lowered fusion barrier found with $\mathrm{n}$-rich $\mathrm{RNB}$ projectiles (compared to stable beams) allows the formation of evaporation residues (ERs) at lower excitation energies. Finally such n-rich reaction products will have higher survival probabilities.

A simple example should suffice to demonstrate these effects. Consider the reaction of stable ${ }^{32} \mathrm{~S}$ with ${ }^{208} \mathrm{~Pb}$ and radioactive ${ }^{38} \mathrm{~S}$ with ${ }^{208} \mathrm{~Pb}$. The nominal compound nuclei are ${ }^{240} \mathrm{Cf}$ and ${ }^{246} \mathrm{Cf}$ formed at excitation energies of 43.6 and $22.7 \mathrm{MeV}$, respectively (assuming the reactions are carried out at the interaction barriers of 149.4 and $133.3 \mathrm{MeV}$ ). The predicted ratio of the two evaporation residue production cross sections $\left(\sigma_{38} / \sigma_{32}\right)$ is 8.2 $\times 10^{4}$, i.e., the radioactive beam reaction has an associated production cross section that is five orders of magnitude greater than the stable beam reaction. The product of the ${ }^{208} \mathrm{~Pb}\left({ }^{32} \mathrm{~S}, 4 \mathrm{n}\right)$ reaction, ${ }^{236} \mathrm{Cf}$, is estimated [23] to have a $t_{1 / 2}$ of $9.4 \mathrm{~s}$, while the product of the ${ }^{208} \mathrm{~Pb}\left({ }^{38} \mathrm{~S}, 2 \mathrm{n}\right)$ reaction, ${ }^{244} \mathrm{Cf}$, is known to have $t_{1 / 2}=19.4 \mathrm{~m}$. The longer $t_{1 / 2}$ of the ${ }^{244} \mathrm{Cf}$ can be important when one is studying the chemistry and atomic physics of these nuclei.

RNBs are used primarily in the synthesis of n-rich heavy nuclei using complete fusion reactions. For these reactions the cross section for producing a heavy evaporation residue can be written as a non-separable product of three factors, which express the

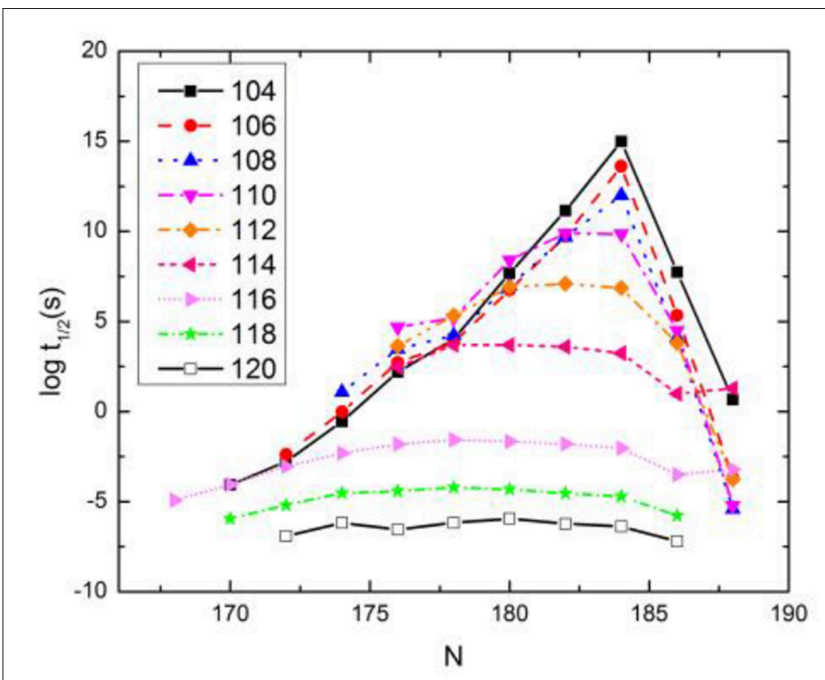

FIGURE 1 | The predicted [22, 23] half-lives of even-even heavy nuclei vs. their neutron number.

capture cross section, $\sigma_{\text {capture, }}$, the fusion probability, $\mathrm{P}_{\mathrm{CN}}$, and the survival probability, $\mathrm{W}_{\text {sur }}$. This equation is

$$
\sigma_{E V R}(E)=\sum_{l=0}^{\infty} \sigma_{\text {Capture }}(E, l) P_{C N}(E, l) W_{\text {sur }}(E, l)
$$

where $\sigma_{\text {capture }}(E, \mid)$ is the capture cross section at center of mass energy $\mathrm{E}$ and spin $l \mid \mathrm{P}_{\mathrm{CN}}$ is the probability that the projectiletarget system will evolve from the contact configuration to inside the fission saddle point to form a completely fused system. $\mathrm{W}_{\text {sur }}$ is the probability that the completely fused system will de-excite by neutron emission rather than fission.

Loveland [25] has evaluated the capture cross sections relevant for heavy element synthesis and finds that the capture cross sections are known to within a factor of two. There is a wellestablished formalism to calculate the survival probabilities, where the principal uncertainty is the values of the fission barrier heights used in these calculations. Loveland [25] concluded that $\mathrm{W}_{\text {sur }}$ is known within a factor 3 . In the same paper, Loveland concluded that $\mathrm{P}_{\mathrm{CN}}$ is known within an order of magnitude.

We can apply this knowledge about heavy element complete fusion cross sections to the problem of making new heavy nuclei with radioactive beams. The calculational framework is a brute force technique [2] in which one starts with the projected $\mathrm{RNB}$ intensities at various radioactive beam facilities and then considers all possible combinations of a radioactive projectile with a "stable" target nucleus. One varies the projectile energy and evaluates $\sigma_{\text {EVR }}$. Upon making a reasonable assumption about the target thickness $\left(0.5 \mathrm{mg} / \mathrm{cm}^{2}\right)$, one can evaluate the product yield in atoms/day.

Sources of information about possible radioactive beam intensities have to be carefully examined. The US FRIB project has a website ${ }^{1}$ giving the current best estimates of the radioactive

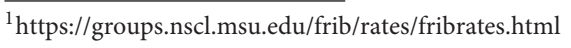


beams available at this facility. At this Projectile Fragmentation facility one has to be careful to distinguish between the fast fragmentation beams and the re-accelerated beams-which are the relevant beams for complete fusion reactions at near barrier energies. Older information about SPIRAL2 can be found [8]. RNB intensities at the ISAC2 facility are available ${ }^{2}$. The US considered a "concept design" of a RNB facility, RIA, which had unrealistically high estimates of possible beam intensities. In a similar vein, the use of exotic target nuclei, like ${ }^{250} \mathrm{Cm}$ needs to be carefully and critically evaluated.

Stable beams are available at intensities of $\sim 3-6 \times 10^{12}$ $\mathrm{p} / \mathrm{s}$ while radioactive beam intensities are substantially less (by 4 orders of magnitude or more). Consequently the heavy element production rates using radioactive beams are usually 3 orders of magnitude less than those achieved with stable beams. Radioactive beams are not a pathway to new superheavy elements $(Z>118)$.

Does this mean that RNBs are useless in synthesizing new nrich heavy nuclei? No, there are "windows of opportunity" for making new n-rich isotopes of elements 104-107 [25]. These reactions generally involve high intensity light beams, like $\mathrm{O}, \mathrm{Ne}$, $\mathrm{Mg}$, etc. reacting with n-rich heavy targets.

In making these estimates, one must be aware that new technical developments might result in new opportunities for the synthesis of new $\mathrm{n}$-rich heavy nuclei.

What is the future of these efforts to use n-rich RNBs for heavy element synthesis? There are some efforts to develop the use of beams like ${ }^{46} \mathrm{~K}$ at existing facilities like ReA3 to test how well our established formalisms predict the outcome of relevant complete fusion cross section measurements. Wakhle et al. [4] studied the near-barrier fusion of stable ${ }^{39} \mathrm{~K}$ and radioactive ${ }^{46} \mathrm{~K}$ with ${ }^{181} \mathrm{Ta}$. They found enhanced capture cross sections with the ${ }^{46} \mathrm{~K}$ projectile compared to the stable ${ }^{39} \mathrm{~K}$. The authors took their results and extrapolated them to the ${ }^{46,47,48} \mathrm{~K}+{ }^{226} \mathrm{Ra}$ and ${ }^{227} \mathrm{Ac}$ reactions. Using current estimates of FRIB beam intensities ${ }^{1}$, they predicted production rates of $\mathrm{n}$-rich isotopes of $\mathrm{Bh}$ and Hs to be $\sim 0.1$ atoms/day. Future experiments with the ReA6 accelerator (where beams of RNBs at $6 \mathrm{~A} \mathrm{MeV}$ might be available) may lead to "real" syntheses of n-rich heavy nuclei. Li et al. [10] have shown that the use of light RNBs like ${ }^{22} \mathrm{O}$ can lead to favorable situations for the synthesis of new n-rich isotopes of No.

\section{MNT REACTIONS-GENERAL}

As mentioned previously, MNT reactions are thought to be a pathway to new n-rich heavy nuclei with $Z \leq 118$. The initial investigations of these reactions at GSI and LBNL [11-16] were not very promising, but recent calculations of Zagrebaev and Greiner [18-20] have invigorated research in this area. However, these reactions are difficult to study because of the low intensities of the heavy beams used in these reactions, the predicted low cross sections for these reactions (pb-nb) and the difficulty of detecting the most $\mathrm{n}$-rich products, which are $\beta$-emitters. [A recent review article summarizes the situation [26]].

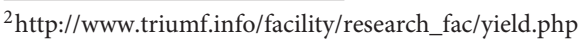

There have been several new initiatives involving MNT reactions to make n-rich nuclei. To begin with, there is a long-standing program to use MNT reactions to make nuclei for nuclear spectroscopy [27-32]. More recently there has been interest in using MNT reactions to make nuclei near the $N=$ 126 neutron shell closure (to study r-process nucleosynthesis). This effort has culminated in a large project at the Argonne National Laboratory, the $N=126$ factory. In the $N=126$ factory, an intense beam $(5 \mathrm{pmA})$ of $9 \mathrm{~A} \mathrm{MeV}{ }^{136} \mathrm{Xe}$ is used to bombard a rotating ${ }^{198} \mathrm{Pt}$ target. The recoiling reaction products are stopped in a gas catcher, mass separated and transferred to nuclear spectroscopy stations for further study. In addition to this project, there have been a number of studies focused on the yields of $N=126$ products in MNT reactions [7, 20, 33-39] (Zhu, Personal communication). A central issue has emerged from these studies, i.e., what are the optimum strategies for making $N=126$ isotones in MNT reactions? Should one focus on the grazing collisions where the transfer products are emitted at the grazing angle for reactions like ${ }^{136} \mathrm{Xe}+{ }^{198} \mathrm{Pt}$ or should one look at near central collisions where the products are emitted near $0^{\circ}$ ? Experiments are underway to address this issue directly for the ${ }^{136} \mathrm{Xe}+{ }^{198} \mathrm{Pt}$ reaction (Zhu, Personal communication). Zagrebaev and Greiner have clearly stated [20] that the relevant collisions for producing heavy $\mathrm{n}$-rich transfer products are the near central (deep inelastic) collisions (DIC). Karpov and Saiko [37] reinforce this conclusion stating "At lower near-barrier energies, even central collisions lead to low excitation energies of primary products." This suggestion to look for interesting transfer products at small angles has been reinforced by recent experiments at the velocity filter SHIP $[34,40]$ where transfer products from the ${ }^{58,64} \mathrm{Ni}+{ }^{207} \mathrm{~Pb}$ reaction were observed at angles of $0-2^{\circ}$. However, a recent radiochemical study [41] of the ${ }^{48} \mathrm{Ca}+{ }^{248} \mathrm{Cm}$ reaction at a beam energy of $10 \%$ above the Coulomb barrier showed the angular distributions of the Fm isotopes $(\Delta \mathrm{Z}=+4)$ were peaked close to the laboratory grazing angle of $27^{\circ}$.

A process related to multi-nucleon transfer is the quasifission process, described in depth by Hinde et al. [42]. Quasifission bridges the gap between multi-nucleon transfer reactions where the colliding nuclei come into sufficiently close contact to exchange nucleons without altering their average mass and the fusion process where the reacting nuclei lose their identity by amalgamating into a compound nucleus. Quasifission processes occur over time scales of 1-10 zs [43] while MNT processes occur on a more rapid time scale.

\section{MNT REACTIONS-EXPERIMENTS}

It is important that we test the various models for MNT reactions, to evaluate their utility in planning future experiments and to gain a correct understanding of how MNT reactions work. There are five competing models or theoretical approaches for describing MNT reactions, the GRAZING model [44-46], the dinuclear systems model (DNS) ${ }^{3}[6,7,10,38,47-53]$, the Improved Quantum Molecular Dynamics model (ImQMD) [54-57], the

$\overline{{ }^{3} \text { http://www.to.infin.it/nanni/grazing }}$ 
Langevin approach of Zagrebaev and Greiner [18], and Karpov and Saito [37] and the time-dependent Hartree-Fock model (TDHF) $[58,59]$ and hybrids of these models.

We have focused our experimental efforts [35, 60,61] on gross tests of these models where we measure the yields of various MNT products and compare our observations with model predictions. The systems we have studied are $5.46 \mathrm{~A}$ $\mathrm{MeV}{ }^{136} \mathrm{Xe}+{ }^{208} \mathrm{~Pb}, 6.67 \mathrm{~A} \mathrm{MeV}{ }^{204} \mathrm{Hg}+{ }^{198} \mathrm{Pt}$, and $5.9 \mathrm{~A}$ $\mathrm{MeV}{ }^{136} \mathrm{Xe}+{ }^{198} \mathrm{Pt}$. These studies have been done at projectile energies ranging from 1.06 to $1.16 \mathrm{~V}_{\mathrm{B}}$, consistent with the idea of preserving shell effects in these collisions.

Barrett et al. [35] reported the measurement of the yields of $235 \mathrm{PLF}$ and TLFs for the reaction of $\mathrm{E}_{\mathrm{c} . \mathrm{m} .}=450 \mathrm{MeV}{ }^{136} \mathrm{Xe}$ $+{ }^{208} \mathrm{~Pb}$. The experiment was done at the Gammasphere facility of the Argonne National Laboratory and utilized thick target measurements of product nuclides measured in beam and postirradiation. This extensive data set (76 TLFs and 159 PLFs and fission fragments) of fragment yields for this reaction has served as a testing ground for models of multinucleon transfer reactions at near barrier energies.

Before doing that comparison, some general conclusions can be drawn from Barrett et al. [35] and other similar studies [33, 34, 62-65]. They are;

(a) The more n-rich the projectile, the more n-rich the TLFs are $[35,40,62]$

(b) The greater the projectile energy for a given reaction, the higher the yields of the n-rich transfer products [35, 65].

(c) $N=126 \mathrm{TLF}$ ranging from $\mathrm{Tl}$ to $\mathrm{Ra}(\Delta \mathrm{Z}=-1$ to $\Delta \mathrm{Z}=+6)$ are made in the ${ }^{64} \mathrm{Ni}+{ }^{207,208} \mathrm{~Pb}$ reactions $[40,62]$

Barrett et al. [35] found that a comparison of their measured $\mathrm{PLF}$ and TLF yields for the ${ }^{136} \mathrm{Xe}+{ }^{208} \mathrm{~Pb}$ reaction with the predictions of the semi-classical GRAZING model ${ }^{3}$ showed that the GRAZING model predictions are adequate for describing then yields of near projectile and near target species, but they grossly underestimate (by orders of magnitude) the yields of all other products. GRAZING is not a model for large proton transfers. For the production of $N=126$ nuclei, GRAZING overestimates the yields of the neutron deficient nuclei but grossly underestimates the yields of the products resulting from large proton transfers.

Zagrebaev and Greiner have suggested an alternate formalism $[18-20,36,66]$ for predicting the yields of MNT reactions at near barrier energies $\left(\mathrm{E}_{\mathrm{c} . \mathrm{m} .}=1.0-1.2 \mathrm{~V}_{\mathrm{B}}\right.$ ). The predictions of Zagrebaev and Greiner when applied to the ${ }^{136} \mathrm{Xe}+{ }^{208} \mathrm{~Pb}$ reaction correctly describe the magnitude and maxima of the observed [35] TLF transfer cross sections for a wide range of transfers $(\Delta \mathrm{Z}=-8$ to $\Delta \mathrm{Z}=+2)$. For $(\Delta \mathrm{Z}=+4)$, the measured TLF yields are four orders of magnitude higher than the predicted maxima.

An extension or generalization of the Langevin approach of Zagrebaev and Greiner $[19,20,36,66]$ to predicting the outcome of MNT reactions is found in the work of Karpov and Saiko [37]. These authors treat the reaction of ${ }^{136} \mathrm{Xe}+{ }^{209} \mathrm{Bi}\left[\mathrm{E}_{\mathrm{c} . \mathrm{m} .}=\right.$ 569,684.and $861 \mathrm{MeV}$ [67-69]], the reaction of ${ }^{136} \mathrm{Xe}+{ }^{208} \mathrm{~Pb}$ $\left[\mathrm{E}_{\mathrm{c.m} .}=450\right.$ [35], 526 and $617 \mathrm{MeV}$ [65]], and the reaction of ${ }^{136} \mathrm{Xe}+{ }^{198} \mathrm{Pt}\left[\mathrm{E}_{\mathrm{c} . \mathrm{m} .}=643 \mathrm{MeV}\right.$ [59]]. These authors conclude that the production yields of neutron-rich nuclei with $N=126$ depend weakly on the incident energy (see discussion below, however, about the ${ }^{136} \mathrm{Xe}+{ }^{198} \mathrm{Pt}$ reaction). As mentioned earlier, they calculate the angular distributions of the MNT products finding the distributions are peaked at the grazing angle for larger energies but are more forward peaked for near barrier collision energies. The calculated and measured total kinetic energy losses, angular and charge distributions are in reasonable agreement for the ${ }^{136} \mathrm{Xe}+{ }^{209} \mathrm{Bi}$ reaction. For the ${ }^{136} \mathrm{Xe}+{ }^{208} \mathrm{~Pb}$ reaction at $\mathrm{E}_{\mathrm{c} . \mathrm{m} .}=450 \mathrm{MeV}$, the Karpov and Saiko calculations are in better agreement with the data [35] for the neutron-deficient TLFs and PLFs but seriously overestimate the yields of the most n-rich products.

Three groups $[6,38,50]$ use the DNS model to predict the yields of the MNT products in the ${ }^{136} \mathrm{Xe}+{ }^{208} \mathrm{~Pb}$ reaction. In the work of [6], the yields of the proton pickup products ( $\mathrm{Tl}, \mathrm{Hg}, \mathrm{Au}$, and $\mathrm{Pt}$ ) are reasonably well described, but the calculations of yields of the trans-target nuclei (Bi, Po, At, and $\mathrm{Rn})$ underestimate the measured cross sections. In the work of [38], the proton pickup product yields are reasonably described with the exception of $\mathrm{Au}$ as are the yields of the trans-target nuclides $\mathrm{Bi}$ and Po but the yields of the larger transfers leading to the production of At and $\mathrm{Rn}$ are underestimated. In the work of Wen et al. [50], a hybrid of the GRAZING and DNS models is used to predict the yields of the MNT products from the ${ }^{136} \mathrm{Xe}+$ ${ }^{208} \mathrm{~Pb}$ reaction. The implication is that the small transfers are the result of grazing collisions while the larger transfers are the result of more central collisions.

The GRAZING, DNS, and ImQMD models were used by [48] to study the ${ }^{136} \mathrm{Xe}+{ }^{208} \mathrm{~Pb}$ reaction. Excellent fits to the transfer product data for nuclides ranging from $\mathrm{Pt}(\Delta \mathrm{Z}$ $=-4)$ to At $(\Delta \mathrm{Z}=+3)$ although the predicted ImQMD distributions are broader than the observed distributions. In these multi-component fits the ImQMD model does the best job of reproducing the observations over a wide range of products.

Another class of models for studying MNT reactions are the time-dependent Hartree-Fock (TDHF) calculations [70-74]. TDHF calculations offer a fully microscopic description of the MNT processes with minimum assumptions on the dynamics. As such, they are free of many of the assumptions used in other models. TDHF theory is expected to be able to describe transfer mechanisms operating at various impact parameters in a unified way.

Sekizawa et al. [70-73] have studied the use of TDHF calculations to describe MNT reactions in a variety of systems. Comparison of the calculations with measured cross sections shows the TDHF calculations describe the cross sections for few nucleon transfers $(|\Delta Z| \leq 2)$ adequately, but as the number of transferred nucleons increases, the agreement gets worse. These workers have shown the importance of including the effect of the de-excitation of the primary fragments by neutron evaporation and fission when comparing the calculations with the measurements. In this regard, when treating very heavy systems, it is important to use "after-burners" that are suitable for situations where most of the primary product nuclei fission [46] rather than generic approaches. 
TDHF calculations were used by Simenel et al. to study MNT in the ${ }^{232} \mathrm{Th}+{ }^{250} \mathrm{Cf}$ reaction at $\mathrm{E}_{\mathrm{c} . \mathrm{m} .}=916 \mathrm{MeV}$. While the deexcitation of the primary MNT products was not considered, the authors were able to conclude that the primary TLFs were more $\mathrm{n}$-rich than those seen in fusion-evaporation reactions.

\section{MNT REACTIONS $-N=126$}

As discussed earlier, one of the exciting applications of MNT reactions is the synthesis of nuclei near the $N=126$ shell closure. Watanabe et al. [59], in a seminal study, measured the yields of several PLFs formed in the $1085 \mathrm{MeV}(8 \mathrm{~A} \mathrm{MeV}){ }^{136} \mathrm{Xe}+{ }^{198} \mathrm{Pt}$ reaction along with the TLFs $\mathrm{Hg}$ and Os. Neutron rich isotopes of Os were formed in collisions involving low total kinetic energy release, while higher total kinetic energy events resulted in the production of neutron deficient isotopes.

Desai et al. [74] extended this work by studying the reaction of $760 \mathrm{MeV}$ (5.59 A MeV) ${ }^{136} \mathrm{Xe}+{ }^{198} \mathrm{Pt}$. At the lower beam energy, the yields of the n-rich TLFs decreased by an order of magnitude or more compared to the data of [59], clearly indicating the importance of using large beam energies in systems where fission decay of the MNT products is not very important.

\section{MNT REACTIONS-HEAVY TRANSFER PRODUCTS}

Another study that is more relevant to the synthesis of new n-rich heavy nuclides is the work of Welsh et al. [60]. These workers studied the formation of MNT products in the near-symmetric collision of $6.67 \mathrm{~A} \mathrm{MeV}{ }^{204} \mathrm{Hg}+{ }^{198} \mathrm{Pt}$. The predictions of the GRAZING model, the DNS model and a hybrid GRAZING/DNS model were compared to the experimental data. The predicted cross sections were a factor of $10-10^{4}$ lower than the observed cross sections. The Improved Quantum Molecular Dynamics (ImQMD) model predictions were also compared to the measurements. The ImQMD predictions were closer to the data, but underestimated the measured yields by factors of 10-100, perhaps due to the lack of shell effects in the ImQMD simulations. This underestimation of the MNT yields is actually encouraging for attempts to synthesize very heavy MNT products.

Gotz et al. [41] were among the first groups in the modern era to experimentally test the ability to form very heavy MNT products. They studied, using radiochemical techniques, the reaction ${ }^{48} \mathrm{Ca}+{ }^{248} \mathrm{Cm}$ at $\mathrm{E}_{\mathrm{lab}}=262 \mathrm{MeV}$. They identified trans Cm nuclides by alpha spectroscopy and focused their efforts on understanding the kinematics of these reactions. For the Fm isotopes (the heaviest trans-target nuclei observed), the angular distributions peaked near the grazing angle, $\theta_{\mathrm{gr}}=27^{\circ}$ and the kinetic energies were low $(\sim 80 \mathrm{MeV})$. This suggests such products are made in grazing collisions, not deep-inelastic collisions. The nuclides with $\mathrm{Z}<\mathrm{Z}_{\text {target }}$ seem to be associated with (DIC) collisions where there is a low survival of the MNT products.

One fascinating set of studies of the synthesis of very heavy nuclei by MNT are the studies of Natowitz et al. [75-78] at
Texas A\&M. In a series of seminal papers from 2011 to 2018, these authors describe the possible formation of very heavy nuclei in MNT reactions. The initial publication of this group [75] described a study of the $7.5 \mathrm{~A} \mathrm{MeV}{ }^{197} \mathrm{Au}+{ }^{232} \mathrm{Th}$ reaction. Using the Big Sol spectrometer, the authors reported the formation of nuclei with $Z \sim 100$ and a production cross section of $\sim 11 \mathrm{nb}$. The authors were cautious and expressed the need for further work to confirm this remarkable result $(\Delta Z=+10)$. Further

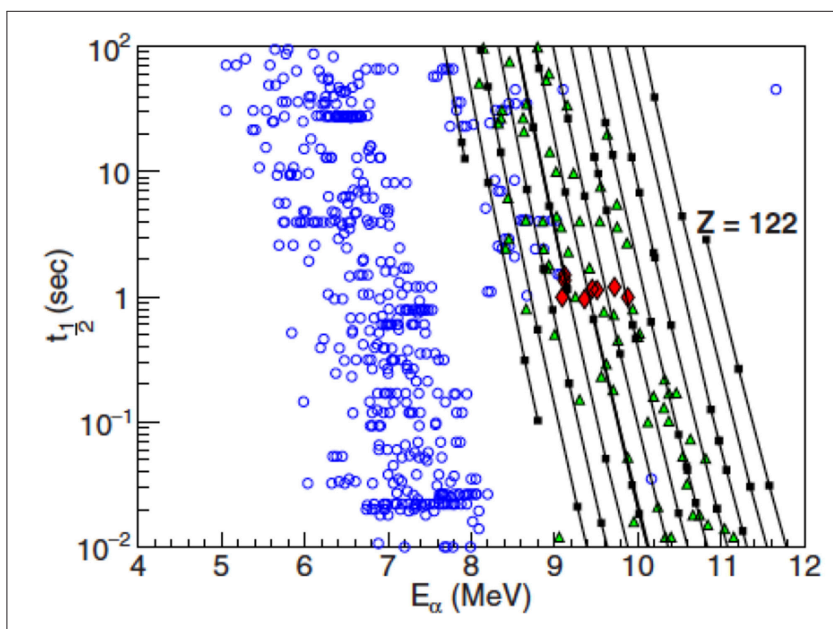

FIGURE 2 | A plot of $t_{1 / 2}$ vs. $E_{\alpha}$ for coincident pairs of TLFs from the ${ }^{238} U+$ ${ }^{232}$ Th reaction from Wuenschel et al. [78]. Open circles denote $Z \leq 101$ and filled triangles indicate $Z \geq 101$. The lines and filled squares indicate the predictions of Agbemava et al. [79] for e-e nuclei with $Z=98-122$. The solid diamonds indicate the experimental data.

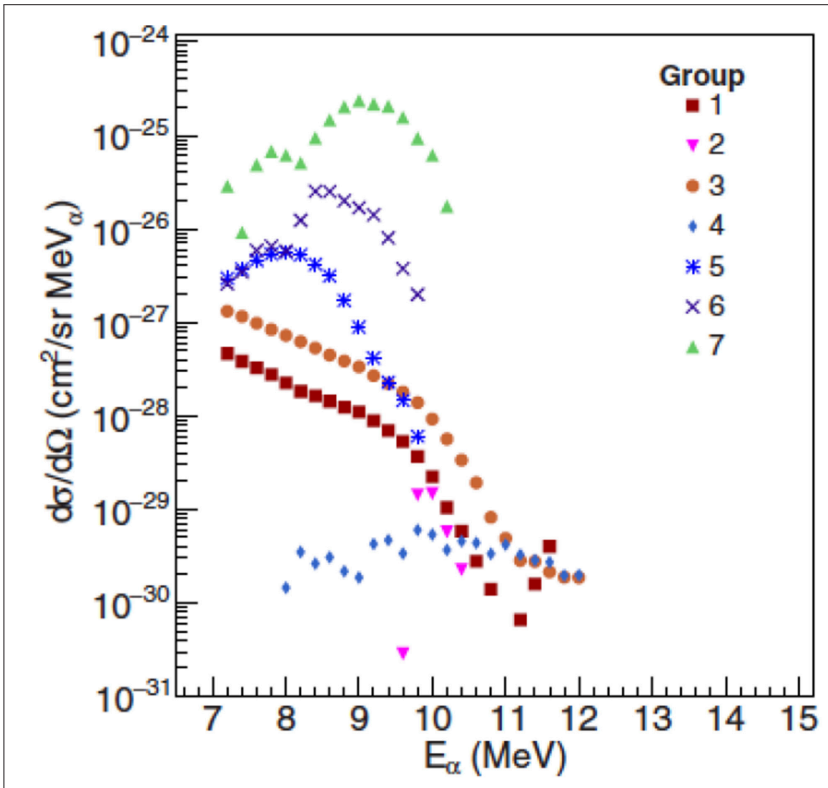

FIGURE 3 | A plot of the differential cross section of TLF events in the angular range from 31 to $51^{\circ}$ for the reaction of ${ }^{238} \cup$ with ${ }^{232} \mathrm{Th}$. Groups 1-7 correspond to various half-life ranges with Group 1 corresponding to the shortest half-lives and Group 7 the longest half-lives. 
details of this experiment were presented in Majka et al. [76] along with plans to use an "active" catcher setup for further studies [77]. Wuenschel et al. [78] reported the results of an investigation of the ${ }^{238} \mathrm{U}+{ }^{232} \mathrm{Th}$ reaction at projectile energies of 6.1-7.5 A MeV. Using an active catcher system with yttrium aluminum perovskite (YAP) to detect transfer products from this reaction, the authors found several interesting events. (The incident beam was degraded from the entrance energy of $7.5 \mathrm{~A}$ $\mathrm{MeV}$ to below the Coulomb barrier $(6.1 \mathrm{~A} \mathrm{MeV})$ in passing through the $11 \mathrm{mg} / \mathrm{cm}^{2}$ Th target). Data was taken with the beam on and with the beam off following the detection of an interesting event. In Figure 2 [78], we see the results of a search for correlated decay pairs. The open circles show events with $Z \leq 101$ and the filled triangles denote $Z>101(\Delta Z>11)$. The systematics [79] of $E_{\alpha}$ vs. $t_{1 / 2}$ for e-e nuclei with $Z=98-122$ is shown as a set of lines. If we restrict our attention to events in the angular range from $31^{\circ}$ to $51^{\circ}$, the differential cross sections of Figure 3 result. (Groups 1-7 correspond to angular range of $31-51^{\circ}$ with further sorting by half-life ranges, with Group 1 corresponding to the shortest half-lives and Group 7 the longest half-lives). The authors conclude that "new activities with $\mathrm{Z}$ as high as 116 are being produced in these reactions." (This corresponds to $\Delta \mathrm{Z}$ $\sim 26$ !!). The quarter point angles for the TLFs range from 31 to $48^{\circ}$, consistent with the angular ranges plotted in Figure 3.

\section{THE FUTURE}

What is the future of the effort to use MNT reactions to form very heavy neutron-rich nuclei? Part of the answer to this question is the determination of whether one should focus on events where the TLFs are formed near the grazing angle or whether the most interesting (high $+\Delta \mathrm{Z}$ ) events occur at angles near the beam axis (Zhu, Personal communication). Can we use existing vacuum separators like SHIP or the FMA to study these reactions (where detection of the products is restricted currently to small angles)?

The most $n$-rich heavy nuclei are $\beta$-emitters, posing a challenge to experimentalists to detect and identify these nuclei. The $N=126$ factory project at the Argonne National Laboratory, described earlier, is one approach to this problem. However, if the interesting n-rich nuclei are formed at small angles with respect to the beam, this approach is problematic.

A stable beam facility that should play a role in future studies of heavy element synthesis reactions is the $S^{3}$ project art GANIL. The GANIL facility, SPIRAL2, is expected to have very high intensity stable ion beams [8]. Two complementary detection systems, SIRIUS and REGLISS, are being developed to detect reaction products. SIRIUS is a highly segmented focal plane detector to detect the implantation and decay of reaction products [80]. REGLIS involves a gas cell for stopping the reaction products followed by resonant ionization of the stopped atoms [80].

\section{REFERENCES}

1. Olsen E, Nazarewicz W. Alpha decay energies of superheavy nuclei: systematic trends. Phys Rev C. (2019) 99:014317. doi: 10.1103/PhysRevC.99.014317
One of the most developed schemes to study MNT reactions that produce very $n$-rich, $\beta$-emitting products is the KEK Isotope Separation System (KISS) [81-83]. This system is designed to study MNT reactions between heavy targets and heavy projectiles. The KISS consists of a dipole magnet and an argon gas-cell with laser resonant ionization. Isotope separation is achieved in two steps; laser resonant ionization to identify the $\mathrm{Z}$ of the reaction product followed by $\mathrm{M} / \mathrm{q}$ separation in a magnetic field. First applications of this system are projected to study nuclei near the $N=126$ shell.

While all these new developments in studying MNT reactions are exciting, one should not forget the use of "conventional" apparatus to study MNT reactions, especially those events produced at small angles with respect to the incident beam, the so-called "deep inelastic collisions." The velocity filter SHIP has been employed in a number of these studies [34, 39-41].

Another "conventional" device to study MNT reactions is the PRISMA spectrometer at Legnaro, Italy. This spectrometer, when operated in coincidence with a time of flight arm, allows identification of the A, Z, and velocity of the PLFs and TLFs. This system has been used in several studies of MNT reactions.

\section{SUMMARY}

What have we learned about and what is the future outlook for forming new very heavy n-rich nuclei? Using facilities like FRIB, SPIRAL2, and TRIUMF, it appears that there are reasonable opportunities to make new n-rich nuclei with $Z=103-108$. The situation, with respect to MNT reactions, is less clear as we have several unanswered experimental questions. Assuming the cross sections are high enough and the facilities to study and identify the reaction products are developed, it may be that MNT reactions are the most promising for producing and studying these nuclei.

\section{DATA AVAILABILITY}

All datasets generated for this study are included in the manuscript and/or the supplementary files.

\section{AUTHOR CONTRIBUTIONS}

The author confirms being the sole contributor of this work and has approved it for publication.

\section{FUNDING}

This work was supported in part by the U.S. National Science Foundation under award 1505043-PHY and the U. S. Department of Energy, Office of Science, Office of Nuclear Physics under award DE-SC0014380.

2. Loveland W. Synthesis of transactinide nuclei using radioactive beams. Phys Rev C. (2007) 76:014602. doi: 10.1103/PhysRevC.76.014612

3. Loveland W. Comment on "Possibility of synthesizing a magic superheavy nucleus". Phys Rev C. (2007) 75:069801. doi: 10.1103/PhysRevC.75.069801 
4. Wakhle A. Hammerton K, Kohley Z, Stiefel K, Walshe J, Jeung DJ, et al. Capture cross sections for the synthesis of new heavy nuclei using radioactive beams. Phys Rev C. (2018) 97:021602. doi: 10.1103/PhysRevC.97. 021602

5. Mun M-H, Adamian GG, Antonenko NV, Lee Y-O. Possibilities of production of neutron-rich Md isotopes in multi-nucleon transfer reactions. Eur Phys J A. (2016) 52:363. doi: 10.1140/epja/i2016-16363-y

6. Zhu L, Su J, Xie W-J, Zhang F-S. Production of neutron-rich transcalifornium nuclei in ${ }^{238} \mathrm{U}$-induced transfer reactions. Phys Rev C. (2016) 94:054606. doi: 10.1103/PhysRevC.94.054606

7. Zhu L, Su J, Xie W-J, Zhang F-S. Theoretical study on production of heavy neutron-rich isotopes around the $\mathrm{N}=126$ shell closure in radioactive beam induced transfer reactions. Phys Lett B. (2017) 767:437. doi: 10.1016/j.physletb.2017.01.082

8. Gales S. SPIRAL2 at GANIL, a world leading ISOL facility at the dawn of the next decade. Prog Part Nucl Phys. (2007) 59:22. doi: 10.1016/j.ppnp.2006.12. 021

9. Zhang G, Li C, Wen P-W, Li J-J, Xu X-X, Li B, et al. Production of neutronrich ${ }^{209-212}$ Pt isotopes based upon a dinuclear systems model. Phys Rev C. (2018) 98:014613. doi: 10.1103/PhysRevC.98.014613

10. Li J, Li C, Zhang G, Zhu L, Liu Z, Zhang F-S. Production cross sections of neutron-rich ${ }^{261-263}$ No isotopes. Phys Rev C. (2017) 95:054612. doi: 10.1103/PhysRevC.95.054612

11. Hulet EK, Lougheed RW, Wild JF, Landrum JH, Stevenson PC, Ghiorso A, et al. Search for superheavy elements in the bombardment of ${ }^{248} \mathrm{Cm}$ with ${ }^{48} \mathrm{Ca}$, Phys Rev Lett. (1977) 39:385. doi: 10.1103/PhysRevLett.39.385

12. Essel H, Hartel K, Henning W, Kienle P, Korner HJ, Rehm KE, et al. Charge and mass transfer in the reaction ${ }^{136} \mathrm{Xe}+{ }^{208} \mathrm{~Pb}$ at energies close to the coulomb barrier. Z Phys A. (1979) 289:265. doi: 10.1007/BF01415787

13. Freisleben H, Hildenbrand KD, Puhlhofer F, Schneider WFW, Bock R, Harrach Dv, et al. The reaction ${ }^{238} \mathrm{U}+{ }^{238} \mathrm{U}$ at $7.42 \mathrm{MeV} / \mathrm{u}$. Z Phys A. (1979) 292:171-89.

14. Schadel M, Brüchle W, Gäggeler H, Kratz JV, Sümmerer K, Wirth G, et al. Actinide production in collisions of ${ }^{238} \mathrm{U}$ with ${ }^{248} \mathrm{Cm}$. Phys. Rev. Lett. (1982) 48:852. doi: 10.1103/PhysRevLett.48.852

15. Moody KJ, Lee D, Welch RB, Gregorich KE, Seaborg GT, Lougheed RW, et al. Actinide production in reactions of heavy ions with ${ }^{248} \mathrm{Cm}$. Phys Rev C. (1986) 33:1315. doi: 10.1103/PhysRevC.33.1315

16. Welch RB, Moody KJ, Gregorich KE, Lee D, Seaborg GT. Dependence of actinide production on the mass number of the projectile: $\mathrm{Xe}+{ }^{248} \mathrm{Cm}$. Phys Rev C. (1987) 35:204-12. doi: 10.1103/PhysRevC.35.204

17. Kratz JV, Loveland W, Moody KJ. Synthesis of transuranium isotopes with atomic numbers $\mathrm{Z} \leq 103$ in multi-nucleon transfer reactions. Nucl. Phys. A. (2015) 944:117-157. doi: 10.1016/j.nuclphysa.2015.06.004

18. Zagrebaev VI, Greiner W. Production of new heavy isotopes in lowenergy multinucleon transfer reactions. Phys Rev Lett. (2008) 101:122701. doi: 10.1103/PhysRevLett.101.122701

19. Zagrebaev VI, Greiner W. Low-energy collisions of heavy nuclei: dynamics of sticking, mass transfer and fusion. J Phys G. (2007) 34:1. doi: 10.1088/0954-3899/34/1/001

20. Zagrebaev VI, Greiner W. Production of heavy and superheavy neutron-rich nuclei in transfer reactions. Phys Rev C. (2013) 83:054615. doi: 10.1103/PhysRevC.83.044618

21. Bass R. Nucleus-nucleus potential deduced from experimental fusion cross sections. Phys Rev Lett. (1977) 39:265-8. doi: 10.1103/PhysRevLett.39.265

22. Smolanczuk R. Properties of the hypothetical spherical superheavy nuclei. Phys Rev C. (1997) 56:812. doi: 10.1103/PhysRevC.56.812

23. Moller P, Nix JR, Kratz KL. Nuclear properties for astrophysical and radioactive-ion-beam applications. At Data Nucl Data Tables. (1997) 66:131. doi: 10.1006/adnd.1997.0746

24. Loveland W, Peterson D, Vinodkumar AM, Sprunger PH, Shapira D, Liang JF, et al. Fusion enhancement in the ${ }^{38} \mathrm{~S}+{ }^{208} \mathrm{~Pb}$ reaction. Phys Rev C. (2006) 74:044607. doi: 10.1103/PhysRevC.74.044607

25. Loveland W. Synthetic paths to the heaviest elements. J Phys Conf Series. (2013) 420:012004. doi: 10.1088/1742-6596/420/1/012004

26. Zhang F-S, Li C, Zhu L, Wen P. Production cross sections for exotic nuclei with multinucleon transfer reactions. Front Phys. (2018) 13:132113. doi: 10.1007/s11467-018-0843-6
27. Wheldon C, Narro JG, Pearson CJ, Regan PH, Podolyak Zs, Warner $\mathrm{DD}$, et al. Yrast states in ${ }^{194}$ Os: the prolate-oblate transition region. Phys Rev C. (2000) 63:011304. doi: 10.1103/PhysRevC.63. 011304

28. Valiente-Dobon JJ, Wheldon C, Regan PH, Wu CY, Cline D, Andreoiu C, et al. Population of ${ }^{195}$ Os via a deep-inelastic reaction, AIP Conf Proc. (2004) 726:249. doi: 10.1063/1.1805958

29. Valiente-Dobon JJ, Regan PH, Wheldon C, Wu CY, Yoshinaga N, Higashiyama $\mathrm{K}$, et al. ${ }^{136} \mathrm{Ba}$ studied via deep-inelastic collisions: Identification of the $\left(\nu \mathrm{h}_{11 / 2}\right)^{-2} 10+$ isomer. Phys Rev C. (2004) 69:024316. doi: 10.1103/PhysRevC.69.024316

30. Wheldon C, Valiente-Dobón JJ, Regan PH, Pearson CJ, Wu CY, Smith JF, et al. Multi-quasiparticle states in ${ }^{184} \mathrm{~W}$ via mutli-nucleon transfer. Eur Phys J A. (2004) 20:365. doi: 10.1140/epja/i2003-10189-8

31. Wheldon C, Valiente-Dobon JJ, Regan PH, Pearson CJ, Wu CY, Macchiavelli AO, et al. Observation of an isomeric state in ${ }^{197}$ Au. Phys Rev C. (2006) 74:027303. doi: 10.1103/PhysRevC.74.027303

32. Vogt A, Birkenbach B, Reiter P, Siciliano M, Hadynska-Klek K, ValienteDobón JJ, et al. Isomers and high-spin structures in the $\mathrm{N}=81$ isotones ${ }^{135} \mathrm{Xe}$ and ${ }^{137} \mathrm{Ba}$. Phys Rev C. (2017) 95:024316. doi: 10.1103/PhysRevC.95. 024316

33. Watanabe $Y X$, Hirayama $Y$, Imai N, Ishiyama H, Jeong SC, Miyatake H, et al. Study of collisions of ${ }^{136} \mathrm{Xe}+{ }^{198} \mathrm{Pt}$ for the KEK isotope separator. NIM B. (2013) 317:752. doi: 10.1016/j.nimb.2013.04.036

34. Beliuskina O, Heinz S, Zagrebaev V, Comas V, Heinz C, Hofmann $\mathrm{S}$, et al. On the synthesis of neutron-rich isotopes along the $\mathrm{N}=126$ shell in multinucleon transfer reactions. Eur Phys J A. (2014) 50:161. doi: 10.1140/epja/i2014-14161-3

35. Barrett JS, Loveland W, Yanez R, Zhu S, Ayangeakaa AD, Carpenter MP, et al. ${ }^{136} \mathrm{Xe}+{ }^{208} \mathrm{~Pb}$ reaction: a test of models of multinucleon transfer. Phys Rev C. (2015) 91:064615. doi: 10.1103/PhysRevC.91.064615

36. Zagrebaev VI, Greiner W. Production of exotic nuclei in low-energy multi-nucleon transfer reactions. Yuanzihe wuli pinglun. (2015) 32:137. doi: 10.11804/NuclPhysRev.32.02.137

37. Karpov AV, Saiko VV. Modeling near-barrier collisions of heavy ions on a Langevin-type approach. Phys Rev C. (2017) 96:024618. doi: 10.1103/PhysRevC.96.024618

38. Feng ZQ. Production of neutron-rich isotopes around $\mathrm{N}=126$ in multinucleon transfer reactions. Phys Rev C. (2017) 95:024615. doi: 10.1103/PhysRevC.95.024615

39. Heinz S. Multinucleon transfer reactions-a pathway to new heavy and superheavy nuclei? In: IOP Conference. Series: Journal of Physics: Conference. Series 1014 012005, Darmstadt (2018).

40. Comas VF, Heinz S, Hofmann S, Ackermann D, Heredia JA, Heßberger FP, et al. Study of multi-nucleon transfer reactions in ${ }^{58,64} \mathrm{Ni}+{ }^{207} \mathrm{~Pb}$ collisions at the velocity filter SHIP, Eur Phys J A. (2013) 49:112. doi: 10.1140/epja/i2013-13112-x

41. Gotz M. Götza S, Kratz JV, Düllmann E, Mokry Ch, Runke J, et al. Radiochemical study of the kinematics of multi-nucleon transfer reactions in ${ }^{48} \mathrm{Ca}+{ }^{248} \mathrm{Cm}$ collisions $10 \%$ above the Coulomb barrier. Nucl Phys A. (2017) 961:1. doi: 10.1016/j.nuclphysa.2017. 02.006

42. Hinde DJ, Jeung DY, Prasad E, Wakhle A, Dasgupta M, Evers M, et al. Sub-barrier quasifission in heavy element formation reactions with deformed actinide target nuclei. Phys Rev C. (2018) 97:024616. doi: 10.1103/PhysRevC.97.024616

43. Knyazheva GN, Itkis IM, Kozulin EM. The time scale of quasifission process in reactions with heavy ions. J Phys Conf Series. (2014) 515:012009. doi: 10.1088/1742-6596/515/1/012009

44. Winther A. Grazing reactions in collisions between heavy nuclei. Nucl Phys A. (1994) 572:191. doi: 10.1016/0375-9474(94)90430-8

45. Winther A. Dissipation, polarization, and fluctuation in grazing heavy-ion collisions and the boundary to the chaotic region. Nucl Phys A. (1995) 594:203.

46. Yanez R, Loveland W. Predicting the production of neutron-rich heavy nuclei in multinucleon transfer reactions using a semi-classical model including evaporation and fission competition, GRAZING-F, Phys Rev C. (2015) 91:044608. doi: 10.1103/PhysRevC.91.044608 
47. Zhu L, Feng ZQ, Zhang FS. Production of heavy neutron-rich nuclei in transfer reactions within the dinuclear systems model. J Phys G Nucl Part Phys. (2015) 42:1. doi: 10.1088/0954-3899/42/8/085102

48. Li C, Zhang F, Li J, Zhu L, Tian L, Wang N, et al. Multi-nucleon transfer in the ${ }^{136} \mathrm{Xe}+{ }^{208} \mathrm{~Pb}$ reaction. Phys Rev C. (2016) 93:014618. doi: 10.1103/PhysRevC.93.014618

49. Zhu L, Su J, Zhang F-S, Theoretical predictions on production of neutrondeficient nuclei with $Z \geq 93$ in multinucleon transfer reactions. Nucl Phys A. (2017) 964:93-9. doi: 10.1016/j.nuclphysa.2017.05.063

50. Wen P-w, Li C, Zhu C, Lin C-j, Zhang F-S. Mechanism of multinucleon transfer reaction based on the GRAZING model and DNS model. J. Phys G Nucl Part Phys. (2017) 44:115101. doi: 10.1088/1361-6471/aa8b07

51. Zhu L, Sun J, Wen P-W. Optimal incident energies for production of neutrondeficient actinide nuclei in the reaction ${ }^{58} \mathrm{Ni}+{ }^{238} \mathrm{U}$, Phys Rev C. (2017) 95:044608. doi: 10.1103/PhysRevC.95.044608

52. Bao X-J, Guo SG, Li JQ, Zhang HF. Influence of neutron excess of projectile on multinucleon transfer reactions. Phys Lett B. (2018) 785:221. doi: 10.1016/j.physletb.2018.08.049

53. Zhu L. Theoretical study on production cross sections of exotic actinide nuclei in multinucleon transfer reactions. Chin Phys C. (2018) 41:124102. doi: 10.1088/1674-1137/41/12/124102

54. Zhao $\mathrm{K}, \mathrm{Wu} \mathrm{X}, \mathrm{Li} \mathrm{Z}$. Quantum molecular dynamics study of the mass distribution of products in 7.0 A MeV ${ }^{238} \mathrm{U}+{ }^{238} \mathrm{U}$ collisions. Phys Rev C. (2009) 80:054607. doi: 10.1103/PhysRevC.80.054607

55. Zhao K, Li Z, Wang N, Zhang Y, Li Q, Wang Y, et al. Production mechanism of neutron-rich transuranium nuclei in ${ }^{238} \mathrm{U}+{ }^{238} \mathrm{U}$ collisions at near-barrier energies. Phys Rev C. (2015) 92:024613. doi: 10.1103/PhysRevC.92.024613

56. Zhao K, Li Z, Zhang Y, Wang V, Li Q, Shen C, et al. Production mechanism of unknown neutron-rich isotopes in ${ }^{238} \mathrm{U}+{ }^{238} \mathrm{U}$ collisions at near-barrier energy. Phys Rev C. (2016) 94:024601. doi: 10.1103/PhysRevC.94.024601

57. Li C, Wen P, Li J, Zhang G, Li B, Xu X, et al. Production mechanism of new neutron-rich heavy nuclei in the ${ }^{136} \mathrm{Xe}+{ }^{198} \mathrm{Pt}$ reaction. Phys Lett B. (2018) 776:278. doi: 10.1016/j.physletb.2017.11.060

58. Sekizawa K, Yabana K. Time-dependent Hartree-Fock calculations for multinucleon transfer and quasifission processes in the ${ }^{64} \mathrm{Ni}+{ }^{238} \mathrm{U}$ reaction. Phys Rev C. (2018) 93:054616.

59. Sekizawa K, Yabana K. Time-dependent Hartree-Fock calculations for multinucleon transfer processes in ${ }^{40,48} \mathrm{Ca}+{ }^{124} \mathrm{Sn},{ }^{40} \mathrm{Ca}+$ ${ }^{208} \mathrm{~Pb}$ and ${ }^{58} \mathrm{Ni}+{ }^{208} \mathrm{~Pb}$ reactions. Phys Rev C. (2013) 88:014614. doi: 10.1103/PhysRevC.88.014614

60. Welsh T, Loveland W, Yanez R, Barrett JS, McCutchan EA, Sonzogni AA, et al. Modeling multi-nucleon transfer in symmetric collisions of massive nuclei. Phys Lett B. (2017) 771:119. doi: 10.1016/j.physletb.2017.05.044

61. Krolas W, Broda R, Fornal B, Pawlat T, Grawe H, Maier KH, et al. Gamma coincidence study of ${ }^{208} \mathrm{~Pb}+350 \mathrm{MeV}{ }^{64} \mathrm{Ni}$ collisions. Nucl Phys A. (2003) 724:289. doi: 10.1016/S0375-9474(03)01544-6

62. Krolas W, Broda R, Fornal B, Pawłat T, Wrzesinski J, Bazzacco D, et al. Dynamical deformation of nuclei in deep-inelastic collisions: a gamma coincidence study of $130 \mathrm{Te}+275 \mathrm{MeV} 64 \mathrm{Ni}$ and $208 \mathrm{~Pb}+$ $345 \mathrm{MeV} 58 \mathrm{Ni}$ heavy ion reactions. Nucl Phys A. (2010) 832:170. doi: 10.1016/j.nuclphysa.2009.10.159

63. Wilson AN, Beausang CW, Amzal N, Appelbe DE, Asztalos S, Butler PA, et al. High spin states following multinucleon transfer. Eur Phys J A. (2000) 9:183. doi: $10.1007 /$ s100500070035

64. Kozulin E, Vardaci E, Dmitriev SN, Itkis MG, Knyazev AG, Loktev TA, et al. Mass distributions of the system ${ }^{136} \mathrm{Xe}+{ }^{208} \mathrm{~Pb}$ at laboratory energies around the Coulomb barrier: a candidate reaction for the production of neutron-rich nuclei at $\mathrm{N}=126$, Phys Rev C. (2012) 86:044611. doi: 10.1103/PhysRevC.86.044611

65. Wilcke WW, Birkeland JR, Hoover AD, Huizenga JR, Schroeder WU, Viola VE, et al. Bombarding energy dependence of the ${ }^{209} \mathrm{Bi}+{ }^{136}$ Xe reaction. Phys Rev C. (1980) 22:128. doi: 10.1103/PhysRevC.22.128

66. Zagrebaev V, Greiner W. New way for the production of heavy neutron-rich nuclei. J Phys G Nucl Part Phys. (2008) 25:125103. doi: 10.1088/0954-3899/35/12/125103

67. Schroeder WU, Birkeland JR, Huizenga JR, Wolf KL, Viola VE. Mechanisms of very heavy-ion collisions: The ${ }^{209} \mathrm{Bi}+{ }^{136} \mathrm{Xe}$ reaction at $\mathrm{E}_{\mathrm{lab}}=1130 \mathrm{MeV}$, Phys Rep. (1978) 45:301. doi: 10.1016/0370-1573(78)90110-2
68. Wollersheim HJ, Wilcke WW, Birkeland JR, Huizenga JR, Schroeder WU, Freisleben $\mathrm{H}$, et al. ${ }^{209} \mathrm{Bi}+{ }^{136} \mathrm{Xe}$ reaction at $\mathrm{E}_{\mathrm{lab}}=1422 \mathrm{MeV}$, Phys Rev C. (1981) 24:2114. doi: 10.1103/PhysRevC.24.2114

69. Watanabe YX, Kim YH, Jeong SC, Hirayama Y, Imai N, Ishiyama $\mathrm{H}$, et al. Pathway for the production of neutron-rich isotopes around the $\mathrm{N}=126$ shell closure. Phys Rev Lett. (2015) 115:172503. doi: 10.1103/PhysRevLett.115.172503

70. Roy BJ, Pal U, Parmar P, Ramachandran K, Santra S, Mohanty AK, et al. Multinucleon transfer study in ${ }^{208} \mathrm{~Pb}\left({ }^{18} \mathrm{O}, \mathrm{x}\right)$ at energies above the Coulomb barrier. Phys Rev C. (2015) 92:024603. doi: 10.1103/PhysRevC.92.024603

71. Sekizawa K, Yabana K. Time-dependent Hartree-Fock calculations for multinucleon transfer and quasifission processes in the ${ }^{64} \mathrm{Ni}+{ }^{238} \mathrm{U}$ reaction. Phys Rev C. (2016) 93:054616. doi: 10.1103/PhysRevC.93.054616

72. Sekizawa K. Microscopic description of production cross sections including deexcitation effects. Phys Rev C. (2017) 96:014615. doi: 10.1103/PhysRevC.96.014615

73. Simenel C, Golabek C, Kedziora DJ. Actinide collisions for QED and superheavy elements with the time-dependent Hartree-Fock theory and the Balian-Veneroni variational principle. EPJ Web Conf. (2011) 17:09002. doi: 10.1051/epjconf/20111709002

74. Barrett S, Yanez R, Lauritsen T, Sonzogni AA, Harker JL, Chiara CJ, et al. The ${ }^{136} \mathrm{Xe}+{ }^{198} \mathrm{Pt}$ reaction: a test of models of multi-nucleon transfer reactions. arXiv:1505.00257v1. (2015).

75. Barbui M, Hagel K, Natowitz JB, Materna T, Quin L, Chubaryan G, et al. Search for heavy and superheavy systems in ${ }^{197} \mathrm{Au}+{ }^{232} \mathrm{Th}$ collisions near the coulomb barrier. J Phys Conf Series. (2011) 312:082012. doi: 10.1088/1742-6596/312/8/082012

76. Majka Z, Barbui M, Chubaryan G, Griffin H, Lunardon M, Hagel K, et al. Experimental search for super and hyper heavy nuclei at Cyclotron Institute, Texas A\&M University. Acta Physica Polonica. (2014) 45:279-90. doi: 10.5506/APhysPolB.45.279

77. Wielock A, Adamczyk M, Barbui M, Blando N, Giuliani G, Hagel K, et al. A novel approach to the island of stability of super-heavy elements search. EPJ Web Conf. (2016) 117:01003. doi: 10.1051/epjconf/2016117 01003

78. Wuenschel S, Hagel K, Barbui M, Gauthier J, Cao XG, Wada R, et al. An experimental survey of the production of alpha decaying heavy elements in reactions of ${ }^{238} \mathrm{U}+{ }^{232} \mathrm{Th}$ at 7.5-6.1 MeV/nucleon. Phys Rev C. (2018) 97:064602. doi: 10.1103/PhysRevC.97.064602

79. Agbemava SE, Afanasjev AV, Ray D, Ring P. Assessing theoretical uncertainties in fission barriers of superheavy nuclei. Phys Rev C. (2017) 92:054310. doi: 10.1103/PhysRevC.92.054310

80. Dechery F, Savajols H, Authier M, Drouart A, Nolen J, Ackermann D, et al. The super separator spectrometer S3 and the associated detection systems: SIRIUS \& LEB-REGLIS3. Nucl Instru Meth Phys Res B. (2016) 376:125. doi: 10.1016/j.nimb.2016.02.036

81. Kimura S, Ishiyama H, Miyatake H, Hirayama Y, Watanabe YX, Jung HS, et al. Development of the detector system for $\beta$-decay spectroscopy at the KEK Isotope Separation System. Nucl Instru Meth Phys Res B. (2016) 376:338. doi: 10.1016/j.nimb.2016.01.041

82. Hirayama $\mathrm{Y}$, Miyatake $\mathrm{H}$, Watanabe $\mathrm{YX}$, Imai $\mathrm{N}$, Ishiyama $\mathrm{H}$, Jeonget SC. al. Beta-decay spectroscopy of r-process nuclei around $\mathrm{N}=126$. EPJ Web of Conf. (2016) 109:08001. doi: 10.1051/epjconf/2016109 08001

83. Miyatake M, Wada M, Watanabe XY, Hirayama Y, Schury P, Ahmed M, et al. Present Status of the KISS Project. AIP Conf Proc. (2017) 1947:020018. doi: $10.1063 / 1.5030822$

Conflict of Interest Statement: The author declares that the research was conducted in the absence of any commercial or financial relationships that could be construed as a potential conflict of interest.

Copyright (c) 2019 Loveland. This is an open-access article distributed under the terms of the Creative Commons Attribution License (CC BY). The use, distribution or reproduction in other forums is permitted, provided the original author(s) and the copyright owner(s) are credited and that the original publication in this journal is cited, in accordance with accepted academic practice. No use, distribution or reproduction is permitted which does not comply with these terms. 